Suppose every set of $n-k$ congruences of the orthogonal ennuple is normal to a family $V_{k}$. Then equations (10) are satisfied when $h, i$ and $j$ take on any distinct values. But Ricci has shown that $\gamma_{h i j}=-\gamma_{i h j}$; combining, we find $\gamma_{h i j}=0$ for $h, i, j$ distinct, and this is a sufficient condition that all the congruences be normal. Consequently, if every set of $n-k$ congruences $(k>1)$ of an orthogonal ennuple has a family of $k$ dimensional hypersurfaces as orthogonal trajectories, then all of the congruences are normal.

Princeton University

\title{
LIMITS FOR ACTUAL DOUBLE POINTS OF SPACE CURVES*
}

BY T. R. HOLLCROFT

1. Introduction. Noethert has proved that space curves of maximum genus on non-singular surfaces always exist. In the first part of this paper, such curves will be assumed to exist also when any number of actual double points are added, subject only to the fact that the genus can not be negative for a proper curve and to certain other limitations. The purpose of this paper is to ascertain and define these limitations when all of the actual double points of the space curve are cusps or when all are nodes, and to discuss the existence of space curves with any number of actual double points up to and including the maximum.

From Noether's formula for the maximum genus $\pi_{\mu}$ of a curve of order $n$ on a non-singular surface of order $\mu$, is obtained the theorem: The minimum number of apparent double points $h_{\mu}$ for a space curve of order $n$ cut out by

* Presented to the Society, February 24, 1923.

$\dagger$ M. Noether, Zur Grundlegung der Theorie der Algebraischen Raumkurven, AbHandlungen der Preussischen Akademie DeR Wissenschaften, 1882, Section 6. 
a surface of order $\nu$ on a non-singular surface of order $\mu$, if $\mu \nu \geqq n>\mu(\nu-1), \mu \leqq \nu$, is

$$
h_{\mu}=\frac{1}{2}(2 n-\mu \nu)(\mu-1)(\nu-1) .
$$

2. Limits Obtained by Projection. From an arbitrary point, the apparent double points of a space curve project into plane nodes, but from a point on the developable, from a point on the nodal curve of the developable, or from a triple point of this curve, one, two or three apparent double points respectively are projected into cusps on the plane curve of projection. For $n>3$ the nodal curve always exists and for $n \geq 8$ it always has triple points.

Let $C_{n}$ represent a space curve of order $n$ and maximum genus $\pi_{\mu}$ with $h_{\mu}$ apparent double points and lying on a non-singular surface of order $\mu$. Let $C_{n}$ be projected into a plane curve $\bar{C}_{n}$, for $n \leqq 7$, from any point of the nodal curve of the developable surface. Resulting from the projection of the apparent double points of $C_{n}$, the plane curve $\bar{C}_{n}$ will have two cusps and

$$
\delta_{1}=h_{\mu}-2=\frac{1}{2}(2 n-\mu \nu)(\mu-1)(\nu-1)-2
$$

nodes. For $n \geqq 8$ the projection will be made from a triple point of the nodal curve and the plane curve will then have three cusps and $\delta_{1}=h_{\mu}-3$ nodes.

The maximum number of cusps that may be added to the singularities of this plane curve $\bar{C}_{n}$ is the number of cusps of $\overline{C_{n}}$ that can result from the projection of cusps of $C_{n}$, that is, it gives an upper limit for the number of cusps of the space curve. The solution of this problem for a plane curve is given in a former paper.* Use the above values of $\delta_{1}$ in the limits of $\delta_{1}$ given in the solution of this problem, denote the maximum number of cusps of $C_{n}$ by $\beta_{\mu}$ and we have the result:

* T. R. Hollcroft, Singularities of curves of given order, this Bulletin, vol. 29 (1923), Problem 1, pp. 409-10. 
The maximum number of cusps $\beta_{\mu}$ for $C_{n}$ as defined above, provided that $\mu \nu \geqq n>\mu(\nu-1)$, is as follows:

For $n \leqq 7$,

$$
\beta_{\mu}=\frac{1}{2}(n-1)(n-2)-h_{\mu} ;
$$

For $n \geq 8$,

$$
\text { If } h_{\mu} \geq \frac{1}{2} n(n-10)+3+2 \sqrt{4 n+13,}
$$

$$
\left[\beta_{\mu}\right]^{*} \leqq \frac{3}{8}\left[n(n-2)-2\left(h_{\mu}+1\right)\right]
$$

$$
\text { If } \begin{aligned}
\frac{1}{2} n(n-10)+3+2 \sqrt{4 n+13} & >h_{\mu}>\frac{1}{2} n(n-13) \\
& +14-\sqrt{16 n-23,}
\end{aligned}
$$

(3) $\left[\beta_{\mu}\right] \geqq \frac{1}{6}\left[2 n(n-1)-4 h_{\mu}-15\right.$

$$
\left.-\sqrt{4 n(n-9)-8 h_{\mu}+105}\right] \text {; }
$$

If $h_{\mu} \leqq \frac{1}{2} n(n-13)+14-\sqrt{16 n-23}$,

$$
\left[\beta_{\mu}\right] \leqq \frac{1}{4}\left[(n-3)(n+6)-2\left(h_{\mu}+3\right)\right] .
$$

An exception to (1) occurs when $\mu=2$ since a $C_{7}$ on a quadric can have but five cusps.

Since $\mu \nu \geqq n>\mu(\nu-1)$, in (2)

$$
h_{\mu} \geqq \frac{1}{2} n(n-10)+3+2 \sqrt{4 n+13}
$$

only for $\mu+\nu \leqq 8$; and in (3)

$$
h_{\mu} \geqq \frac{1}{2} n(n-13)+14-\sqrt{16 n-23}
$$

only for $\mu+\nu \leqq 14$.

If in the formula for $h_{\mu}$ we let $\mu=2$ and $\nu=n / 2$ or

* The symbol $[x]$ followed by $\leqq(\geqq)$ means the largest (one greater than the largest if not equal) integer contained in the expression on the right. 
$(n+1) / 2$ we obtain $h_{2}=\frac{1}{4} n(n-2)$ or $\frac{1}{4}(n-1)^{2}$ for $n$ even or odd respectively. The inequality

$$
\left[h_{2}\right] \geqq \frac{1}{4} n(n-2)
$$

defines the smallest number of apparent double points for a proper space curve of order $n *^{*}$ Then when $\mu=2$, $C_{n}$ can have the largest number of actual double points possible for a given order $n$.

Using the above value of $h_{2}$ in the preceding limits, we can determine the values of $n$ for which the several limits of $\delta_{1}$ hold and thus obtain the following formulas. For $n \leqq 6$,

$$
\left[\beta_{2}\right] \leq \frac{1}{4}(n-2)^{2} ;
$$

For $7 \leqq n \leqq 11$,

$$
\left[\beta_{2}\right] \leqq \frac{3}{16}\left(n^{2}-2 n-4\right) ;
$$

For $12 \leqq n \leqq 25$,

$$
\left[\beta_{2}\right] \geq \frac{1}{6}\left(n^{2}-15-\sqrt{2 n(n-12)+105}\right) ;
$$

For $n \geqq 26$,

$$
\left[\boldsymbol{\beta}_{2}\right] \leqq \frac{1}{8}\left(n^{2}+8 n-44\right) \text {. }
$$

With the exception of the limit for $n \leqq 6$, these are only upper bounds for $\beta_{2}$ and can not be attained, as will be shown in the two following sections.

Since every rational plane curve of order $n \geqq 4$ may have three or more cusps, the above considerations do not limit the nodes of a space curve.

3. Limits given by $\gamma \geqq 0$. Certain limits for $\beta$ arise from the fact that none of the characteristic numbers of a

* G. Halphen, Sur quelques propriétés des courbes gauches algébriques, Bulletin DE LA Soctété DE France, vol. 2, p. 42. 
proper space curve can be negative, and in some cases these limits are more restrictive than those derived above by projection. Of these, the lowest limit for $\beta$ is given by $\gamma$, the number of cusps of the nodal curve. For $\gamma \geq 0$, there results the inequality

$$
[\beta] \leqq \frac{1}{6}[r(n-6)+4(n-\omega-H)-2 v],
$$

wherein $r$ is the rank, $v$ the number of linear inflections, $\omega$ the number of double tangents and $H$ the number of nodes of a space curve. When $\omega=v=0$, as may happen for curves of any order, the space curve can have a larger number of cusps. There results

$$
[\beta] \leqq \frac{1}{6}[r(n-6)+4(n-H)]
$$

This serves as an upper bound to $\beta$ for all space curves.

Space curves that lie on a quadric have $[h] \geqq n(n-2) / 4$, so that

$$
[r] \leqq \frac{n^{2}}{2}-3 \beta-2 H
$$

Substitute this limit for $r$ in the foregoing inequality derived from $\gamma \geq 0$ and we obtain

$$
\left[\beta_{2}\right] \leqq \frac{1}{6}[n(n-2)-4 H]
$$

Since, for a maximum $\beta, H=0$ or 1 , the limit becomes

$$
\left[\beta_{2}\right] \leqq \frac{1}{6} n(n-2) \text {. }
$$

If, when $n(n-2)$ is divided by 6 , the remainder is greater than or equal 4, there may be one node. This occurs only when $n$ is of the form $n \equiv 1, \bmod 6$.

This formula gives as small a limit for $\beta_{2}$ for $7 \leqq n \leqq 26$ as those obtained by projection, or a smaller limit.

For curves on cubic surfaces, we find that the inequality $\gamma \geqq 0$ leads to the limit 


$$
\left[\beta_{3}\right] \leqq \frac{1}{9}(n-2)(n+3) .
$$

For $n \leqq 8$, all the actual double points may be cusps. The above limit is more restrictive than those derived by projection for $12 \leqq n \leqq 17$ and gives the same value of $\beta_{3}$ for $n=9,10,11$.

For curves on quartic surfaces, there are no values of $n$ for which the limit derived from $\gamma \geq 0$ gives a smaller limit for $\boldsymbol{\beta}_{4}$ than those given by the projection formulas.

If in the above inequality for $\beta$ in terms of $n$ and $H$, we let $\beta=0$ instead of $H$, we obtain

$$
\left[H_{2}\right] \leqq \frac{1}{4} n(n-2) \text {. }
$$

This limit, however, is always greater than the maximum genus of $C_{n}$ on a quadric, so the fact that $\gamma$ cannot be negative does not restrict the number of nodes when $\beta=0$. The same is true for curves on surfaces of higher order than two.

4. Limits Caused by Adjoint Curve. As was shown by Noether,* the necessary and sufficient condition that a noncomposite plane curve be the projection of a space curve is that the double points of the plane curve which are projections of the apparent double points of the space curve lie on a plane curve of order $n-i-3$, where $i \leqq \mu+\nu-4$ if $\mu \nu=n$ or $i \leqq \mu+\nu-5$ if $\mu \nu>n>\mu(\nu-1)$. This curve of order $n-i-3$ is called an adjoint curve. There are adjoint curves for all values of $i$ less than or equal to the given limits, but since the one for the largest value of $i$ is of least order and thus imposes the greatest number of conditions on $C_{n}$, only that one will be considered here.

First consider curves that are complete intersections of the two surfaces of orders $\mu$ and $\nu$, that is, let $\mu \nu=n$. For such curves the greatest value of $i$ is $\mu+\nu-4$ and the order of the adjoint curve of least order is $(\mu-1)(\nu-1)$.

* M. Noether, loc. cit., $\S 4$, Theorem III. 
The number of conditions necessary to determine this adjoint curve is $\frac{1}{2}(\mu-1)(\nu-1)[(\mu-1)(\nu-1)+3]$. Subtracting this from $h_{\mu}=\frac{1}{2} \mu \nu(\mu-1)(\nu-1)$, we find that there are $(\mu-1)(\nu-1)(\mu+\nu-4) / 2$ double points of $\overline{C_{n}}$ through which the adjoint curve passes gratuitously, and therefore that among the $h_{\mu}$ double points of $\overline{C_{n}}$ there exist $\frac{1}{2}(\mu-1)(\nu-1)(\mu+\nu-4)$ linear relations.

For a sufficiently large $n$, these conditions on $\overline{C_{n}}$ lead to a limit to the number of cusps (and, for a still larger $n$, to the number of nodes) of $C_{n}$. The projections of the $h_{\mu}$. apparent double points of $C_{n}$, since they may be projected into $h_{\mu}-3$ nodes and 3 cusps of $\bar{C}_{n}$, account at most for $h_{\mu}+3$ conditions on $\bar{C}_{n}$. Then the number of degrees of freedom of $\overline{C_{n}}$ is

$$
\begin{aligned}
\frac{1}{2} n(n+3)-h_{\mu}- & 3-\frac{1}{2}(\mu-1)(\nu-1)(\mu+\nu-4) \\
= & \frac{1}{2}[6 n-2+(\mu+\nu)(\mu+\nu-5)],
\end{aligned}
$$

all of which may be absorbed by assigning cusps or nodes to $\bar{C}_{n}$. Lefschetz* has proved that a node accounts for one and a cusp for two invariants, and that for the genus $p \geqq 2$, no more than $\frac{1}{2} n(n+3)-8$ of the $\frac{1}{2} n(n+3)$ conditions necessary to determine a plane curve may be invariants. For the curves $\bar{C}_{n}$ involved here, more than eight metric conditions are given in each case by the linear relations that exist among the double points of $\bar{C}_{n}$ which are projections of the apparent double points of $C_{n}$, so that all the remaining degrees of freedom of $\bar{C}_{n}$ may be absorbed by assigning nodes or cusps to $\bar{C}_{n}$.

An upper limit to the number of cusps of $C_{n}$ is, therefore,

$$
[\beta \mu] \leqslant \frac{1}{4}[2(3 n-1)+(\mu+\nu)(\mu+\nu-5)]
$$

and an upper limit to the number of nodes is

$$
H_{\mu}=3 n-1+\frac{1}{2}(\mu+\nu)(\mu+\nu-5) .
$$

* S. Lefschetz, On the existence of loci with given singularities, Transactions of this Soctety, vol. 14 (1913), pp. 23-41. 
When $C_{n}$ is not a complete intersection, for curves with the minimum number of apparent double points, $\mu \nu>n>\mu(\nu-1)$, $i=\mu+\nu-5$ and the number of linear conditions among the $h_{\mu}$ double points of $\bar{C}_{n}$ is, for $\mu \leqq \nu$,

$$
\frac{1}{6}[i(i+1)(i+2)-(i-\mu+1)(i-\mu+2)(i-\mu+3)]
$$

for $\mu \leqq 4$, and

$$
\begin{aligned}
\frac{1}{6}[i(i+1)(i+2)- & (i-\mu+1)(i-\mu+2)(i-\mu+3) \\
& -(i-\nu+1)(i-\nu+2)(i-\nu+3)],
\end{aligned}
$$

for $\mu \geqq 5$.

An upper limit to the cusps of $C_{n}^{\gamma}$ is found as before by subtracting the sum of each of the foregoing expressions and $h_{\mu}+3$ from $\frac{1}{2} n(n+3)$, which gives, respectively,

$$
\begin{array}{r}
{[\beta \mu] \leqq \frac{1}{4}[(n-1)(n+2)+(\mu+\nu-\mu \nu)(2 n+\mu+\nu-\mu \nu-7)} \\
\left.-\frac{1}{3}(\mu-2)(\mu-3)(\mu-4)\right]
\end{array}
$$

for $\mu \leq 4$, and for $\mu \geqq 5$,

$[\beta \mu] \leqq \frac{1}{4}[(n-1)(n+2)+(\mu+\nu-\mu \nu)(2 n+\mu+\nu-\mu \nu-7)]$.

Since the additional terms in the first limit by which it differs from the second vanish identically for values of $\mu$ within the limit $\mu \leqq 4$ for which the formula holds, the second limit for $\beta \mu$ holds for all values of $\mu, \mu \leqq \nu$ and $\mu \nu>n>\mu(\nu-1)$.

Also as before, the upper limit to the number of nodes of $C_{n}$ for $\mu \leqq \nu$ and $\mu \nu>n>\mu(\nu-1)$ becomes $H_{\mu}=\frac{1}{2}[(n-1)(n+2)+(\mu+\nu-\mu \nu)(2 n+\mu+\nu-\mu \nu-7)]$.

These limits for $\beta_{\mu}$ are more restrictive than projection limit (2) for $\mu+\nu \geqq 9$, but since limit (2) holds only for $\mu+\nu \leqq 8$, it is not superseded by this new limit. 
The new limits are more restrictive than projection limit (3) for $\mu+\nu \geqq 9$. Since projection limit (3) holds for $\mu+\nu \leqq 14$, it is superseded by the new limits for all values of $\mu$ and $\nu$ such that $\mu+\nu \geqq 9$. Projection limit (4) is entirely superseded by the new limits for all values of $\mu$ and $\nu$.

5. Summary of Limits. Finally, for any proper space curve of order $n$ which is formed by the intersection of a surface of order $\nu$ with a non-singular surface of order $\mu$ such that $\mu \nu \geqq n>\mu(\nu-1)$, there exist the following upper limits to the number of cusps it may possess:

When $n \leqq 7 \quad(n \leqq 6$ for $\mu=2)$,

$$
\beta_{\mu}=\frac{1}{2}(n-1)(n-2)-h_{\mu} ;
$$

When $n \geq 8$ and $\mu+\nu \leqq 8$,

For $h_{\mu} \geqq \frac{1}{2} n(n-10)+3+2 \sqrt{4 n+13}$

$$
\left[\beta_{\mu}\right] \leqq \frac{3}{8}\left[n(n-2)-2\left(h_{\mu}+1\right)\right]
$$

For $\frac{1}{2} n(n-10)+3+2 \sqrt{4 n+13}>h_{\mu}>\frac{1}{2} n(n-13)$ $+14-\sqrt{16 n-23}$

$\left[\beta_{\mu}\right] \geqq \frac{1}{6}\left[2 n(n-1)-4 h_{\mu}-15-\sqrt{4 n(n-9)-8 h_{\mu}+105}\right] ;$

When $\mu+\nu \geqq 9$,

If $\mu \nu=n$,

$\left[\beta_{\mu}\right] \leqq \frac{1}{4}[(\mu+\nu)(\mu+\nu-5)+2(3 n-1)]$

If $\mu \nu>n>\mu(\nu-1)$, $\left[\beta_{\mu}\right] \leqq \frac{1}{4}[(n-1)(n+2)+(\mu+\nu-\mu \nu)(2 n+\mu+\nu-\mu \nu-7)]$. 
In the limits derived from the properties of the adjoint curve, if the equality sign holds, the curve $C_{n}$ with the maximum number of cusps can have no nodes. If the inequality sign holds, it may have one, but only one.

Under the same general conditions as above, when $\boldsymbol{\beta}_{\mu}=0$ the following limits exist for the number of nodes $H_{\mu}$ :

When $\mu \nu=n, \mu \leqq \nu$,

For $\mu=2, \nu \geqq 15 ; \mu=3, \nu \geqq 11$; all values of $\mu$ such that $4 \leqq \mu \leqq 7$ and $\mu+\nu \geqq 13$; all values of $\nu \geqq \mu>7$;

$$
H_{\mu}=3 n-1+\frac{1}{2}(\mu+\nu)(\mu+\nu-5)
$$

When $\mu \nu>n>\mu(\nu-1), \mu \leqq \nu$,

For $\mu=2, \nu \geqq 16 ; \mu=3, \nu \geqq 13 ; \mu=4, \nu \geqq 11$ if $\nu$ is of form $(n+1) / 4$ or $(n+2) / 4$, or $\nu \geqq 12$ if $\nu$ is of form $(n+3) / 4 ; \mu=5,6,7, \nu=(n+c) / \mu$, if $c=1, \mu+\nu \geqq 14$ and if $c>1, \mu+\nu \geqq 15$; and for all values of $\nu \geqq \mu>7$;

$$
\begin{aligned}
& H_{\mu}=\frac{1}{2}[(n-1)(n+2) \\
& \quad+(\mu+\nu-\mu \nu)(2 n+\mu+\nu-\mu \nu-7)] .
\end{aligned}
$$

For $\mu=2$ and $n$ even, the adjoint curve is of order $\frac{1}{2}(n-2)$ and $\frac{1}{8}(n-2)(n-4)$ conditions are imposed on the $\frac{1}{4} n(n-2)$ double points of $\bar{C}_{n}$. For $7 \leqq n \leqq 26$ the limit

$$
\left[\beta_{2}\right] \leqq \frac{1}{6} n(n-2)
$$

is lower than that given by the projection limits. The number of conditions imposed by these cusps and nodes on $\bar{C}_{n}$ together with the linear relations existing among them amount to $\left(82 n-5 n^{2}-96\right) / 24$. This is negative for $n \geqq 16$, so that for $n \geqq 16$

$$
\left[\beta_{2}\right] \leqq \frac{1}{6} n(n-2)-\frac{1}{48}\left(5 n^{2}-82 n+96\right)=\frac{1}{16}\left(n^{2}+22 n-32\right) .
$$


The limit for $n$ odd is found in a similar way. These limits can also be obtained by substituting $\mu=2$ and $\nu=n / 2$ or $(n+1) / 2$ respectively in the general limits for $\beta_{\mu}$.

For $\mu=3$ and $12 \leqq n \leqq 17$ the limit

$$
\left[\beta_{3}\right] \leqq \frac{1}{9}(n-2)(n+3)
$$

is lower than that given by the projection limits. By taking $\mu=3$ and $\nu=n / 3,(n+1) / 3,(n+2) / 3$, respectively, and following each through as for $\mu=2$, we find that within these limits for $n$ the above limit is lower also than the limits derived from the conditions imposed on $\overline{C_{n}}$ by the adjoint curve.

For $\mu=3$, the following limits exist.

For $n \leqq 7$,

$$
\left[\beta_{3}\right] \geqq \frac{1}{6}(n-1)(n-2) ;
$$

For $8 \leqq n \leqq 11$,

$$
\left[\beta_{3}\right] \leqq \frac{1}{8}\left(n^{2}-9\right) ;
$$

For $12 \leqq n \leqq 17$,

$$
\left[\beta_{3}\right] \leqq \frac{1}{9}(n-2)(n+3) ;
$$

For $n \geqq 18$,

$$
\begin{aligned}
& \text { If } \nu=\frac{n}{3}, \quad\left[\beta_{3}\right] \leqq \frac{1}{36}\left(n^{2}+57 n-72\right) ; \\
& \text { If } \nu=\frac{n+1}{3},\left[\beta_{3}\right] \leqq \frac{1}{36}\left(n^{2}+65 n-116\right) ; \\
& \text { If } \nu=\frac{n+2}{3},\left[\beta_{3}\right] \leqq \frac{1}{36}\left(n^{2}+61 n-98\right.
\end{aligned}
$$

For $\nu=\frac{n}{3}$ and $n \geqq 33$,

$$
H_{3}=\frac{1}{18}\left(n^{2}+57 n-72\right) ;
$$


For $\nu=\frac{n+1}{3}$ and $n \geqq 38$,

$$
H_{3}=\frac{1}{18}\left(n^{2}+65 n-116\right) ;
$$

For $\nu=\frac{n+2}{3}$ and $n \geqq 37$

$$
H_{3}=\frac{1}{18}\left(n^{2}+61 n-98\right) \text {. }
$$

When $\mu=2$, in view of the fact that space curves with the least number of apparent double points for a given $n$ lie on a quadric, this theorem results:

Every proper space curve with the maximum number of cusps for a given order $n$ lies on a quadric and the maximum for each order is given by the following limits.

(I) For $n \leqq 6$,

$$
\left[\beta_{2}\right] \leqq \frac{1}{4}(n-2)^{2} ;
$$

(II) For $7 \leqq n \leqq 15$,

$$
\left[\beta_{2}\right] \leqq \frac{1}{6} n(n-2) ;
$$

(III) For $n$ even and $n \geqq 16$,

$$
\left[\beta_{2}\right] \leqq \frac{1}{16}\left(n^{2}+22 n-32\right) ;
$$

(IV) For $n$ odd and $n \geqq 17$,

$$
\left[\beta_{2}\right] \leqq \frac{1}{16}\left(n^{2}+24 n-41\right) .
$$

For $n=7$ the curve may be rational, that is, it may have one node in addition to the five cusps. For $n>7$, space curves with the maximum number of cusps for a given $n$ are irrational. For some values of $n$ they may have one node, for others, none.

With regard to nodes, a similar statement may be made, viz., every proper space curve with the maximum number of nodes for a given order $n$ lies on a quadric, and the 
maximum for each order is given by the following limits (no limit except genus for $n<30$ ).

For $n$ even and $n \geqq 30$,

$$
H_{2}=\frac{1}{8}\left(n^{2}+22 n-32\right)
$$

For $n$ odd and $n \geqq 31$,

$$
H_{2}=\frac{1}{8}\left(n^{2}+24 n-41\right) \text {. }
$$

6. Existence of Curves. Since for $n \geqq 4$, plane curves with three cusps and any number of nodes up to the maximum allowed by the genus exist, and since any two surfaces may have as many ordinary contacts as the apparent genus of their curve of intersection, there are no further questions as to the existence of space curves with any number of nodes up to and including the maximum, except those relating to the adjoint curve and they will be considered in the discussion of the existence of space curves with cusps.

The fact proved by Noether that space curves with only apparent double points, of maximum genus and lying on non-singular surfaces always exist has been mentioned above. The addition of cusps requires that the two surfaces containing the $C_{n}$ have that number of stationary contacts. So far as the surfaces are concerned, they may have as many stationary contacts as the apparent genus of $C_{n}$. In regard to $C_{n}$ itself, the addition of cusps reduces all the other characteristic numbers, some very rapidly, notably $\gamma$, which serves as an effective limit on $\beta$. Finally, when the maximum $\beta$ has been so chosen that all these characteristic numbers are non-negative (with the single exception of the genus of the nodal curve, which may be negative, since the nodal curve may be composite when the cuspidal curve is not) there appears to be nothing more appertaining only to the space curve itself to interfere with its existence.

If, however, we attempt to project $C_{n}$ upon a plane when $\beta$ is limited only as above, we find that for $n$ greater than 
certain limits, no proper plane curve exists into which $C_{n}$ may be projected and therefore that $C_{n}$ itself does not exist as a proper space curve. In order that the plane curve of projection exist, two conditions must be satisfiedfirst, the number of cusps and nodes assigned to it must not exceed the maximum number a plane curve of that order can possess, and second, the nodes and cusps of the plane curve which are projections of the apparent double points of the space curve, must lie on the adjoint curve.

The existence of plane curves with any number of cusps up to and including the maximum has been proved by both Lefschetz* and Coolidget for $p \leqq p_{0}$, where $p_{0}$ is the genus associated with a given $n$ on the assumption that the bitangents and inflections are both as near zero as possible. Although lacking a formal proof as yet, the existence of curves for $p>p_{0}$ with any number of cusps up to and including the maximum is practically assured.

In all the preceding, we have used the condition that the double points of the plane curve of projection, which are projections of the apparent double points of the space curve, must lie on an adjoint curve only as the necessary condition that the plane curve be the projection of the space curve. But Noether $\ddagger$ and Valentiner $\S$ have both proved that this condition is also sufficient-that such a plane curve is always the projection of a space curve.

Therefore the existence of space curves with cusps up to and including the maximum as given by the preceding limits is established, if plane curves for $p>p_{0}$ with any number of cusps up to and including the maximum exist.

Wells College

* S. Lefschetz, loc. cit., pp. 33-39.

$\dagger$ J. L. Coolidge, On the existence of curves with assigned singul arities this Bulletin, vol. 28 (1922), pp. 451-55.

† M. Noether, loc. cit., § 3, Theorem I.

$\S$ H. Valentiner, Bidrag til Rumcurvenes Theori, Inaugural Dissertation, Kopenhagen, 1881. 